\title{
Laboreal
}

Volume $8 \mathrm{~N}^{\circ} 1$ | 2012

Género, Atividades e Saúde

\section{Sin tiempos : entre la jornada de trabajo femenino y la maternidad en un contexto laboral flexible}

Sem tempos : entre a jornada de trabalho feminino e a maternidade num contex to laboral flexível

Sans temps : entre la journée de travail féminin et la maternité dans un contexte de travail flexible

No time : between the hours of work and childbearing women in a context flexible working

Constanza Gómez Rubio, Karina Pérez Plaza y Denise Dussert Chervellino

\section{(2) OpenEdition}

Journals

Edición electrónica

URL: http://journals.openedition.org/laboreal/7280

DOI: $10.4000 /$ laboreal. 7280

ISSN: 1646-5237

\section{Editor}

Universidade do Porto

Referencia electrónica

Constanza Gómez Rubio, Karina Pérez Plaza y Denise Dussert Chervellino, « Sin tiempos : entre la jornada de trabajo femenino y la maternidad en un contexto laboral flexible », Laboreal [En línea], Volume $8 \mathrm{~N}^{0} 1$ | 2012, Publicado el 01 julio 2012, consultado el 10 octubre 2019. URL : http:// journals.openedition.org/laboreal/7280; DOI : 10.4000/laboreal.7280

Este documento fue generado automáticamente el 10 octubre 2019.

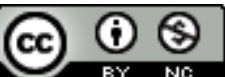

Laboreal está licenciado com uma Licença Creative Commons - Atribuição-NãoComercial 4.0 Internacional. 


\title{
Sin tiempos : entre la jornada de trabajo femenino y la maternidad en un contexto laboral flexible
}

\author{
Sem tempos: entre a jornada de trabalho feminino e a maternidade num \\ contex to laboral flexível
}

Sans temps : entre la journée de travail féminin et la maternité dans un contexte de travail flexible

No time : between the hours of work and childbearing women in a context flexible working

Constanza Gómez Rubio, Karina Pérez Plaza y Denise Dussert Chervellino

\section{NOTA DEL EDITOR}

Manuscrito recibido en : Febrero/2012

Aceptado tras peritaje : Junio/2012

\section{Presentación}

1 En la actualidad, en la mayoría de los países capitalistas de occidente, se desarrolla una fuerte discusión en torno a los variados y marcados cambios que se están produciendo en el mundo del trabajo (Noguera, 2002). Dentro de este contexto, el estudio del trabajo y el empleo requiere de una mirada que vaya más allá de los índices estadísticos y económicos, ubicándolos como construcciones constituidas socialmente, en función de reglas y legitimaciones sociales.

2 Este tipo de discusiones conceptuales, aportan teóricamente a la comprensión y explicación de las transformaciones en el mundo del trabajo. A su vez, pueden ser aplicadas en la práctica, desde una multiplicidad de perspectivas, al trabajo de hombres 
y mujeres concretas (Carrasco y Mayordomo, 2000). De esta manera, la dimensión de género adquiere relevancia como categoría analítica central de los estudios del trabajo y el empleo toda vez que la tensión derivada de la interacción entre al ámbito familiar y laboral, afecta principalmente a las mujeres, reforzando las desigualdades en este ámbito (OIT y PNUD, 2009).

Para cimentar las bases del presente estudio, es conveniente establecer la diferencia entre los términos trabajo y empleo. A pesar de lo polisémico del concepto "trabajo", se entenderá como la actividad de producción de bienes y servicios, con las consecuentes condiciones en que se realiza dicha actividad. Por otra parte, empleo, se puede pensar como las modalidades de acceso y salida del mercado del trabajo, "así como la traducción de la actividad laboral en términos de estatus sociales" (Maruani, 2000, p. 10).

4 En el sistema económico actual, el empleo ocupa un lugar privilegiado. Éste debe ser estudiado desde la perspectiva de las condiciones de circulación y producción de la fuerza de trabajo, rescatando el carácter político, social, y económico de las relaciones de empleo, sin limitarlo, exclusivamente, a la idea de mercado. Por esto, es relevante integrar diversos ámbitos de la realidad social al estudio del empleo, "a fin de confeccionar conceptualmente un sistema que reproduce el sistema que estructura la realidad social" (Prieto, 2000, p. 116). Para una aproximación al concepto de empleo, cabe considerar los siguientes aspectos : empleo como norma social; susceptible a trasformaciones por su carácter socialmente construido ; $y$, la centralidad que adquiere el mismo, en las sociedades modernas (Prieto, 2007).

El lugar del trabajo en las sociedades modernas encuentra su relevancia en tanto modo de jerarquización y clasificación en el orden social. En tal sentido, el trabajo ha adquirido relevancia pública en cuanto a empleo. Por lo que la disposición del orden social es anterior a la consideración del trabajo (Prieto, 2000). En este sentido, Maruani (2000), propone que el estatus social, estructura el modo de empleo, el que a su vez, define el estatus del trabajo. De este modo, el empleo clasifica y segmenta los estatutos del trabajo (Figura 1).

Figura 1 - Jerarquización del empleo y el trabajo en el orden social. Elaboración propria

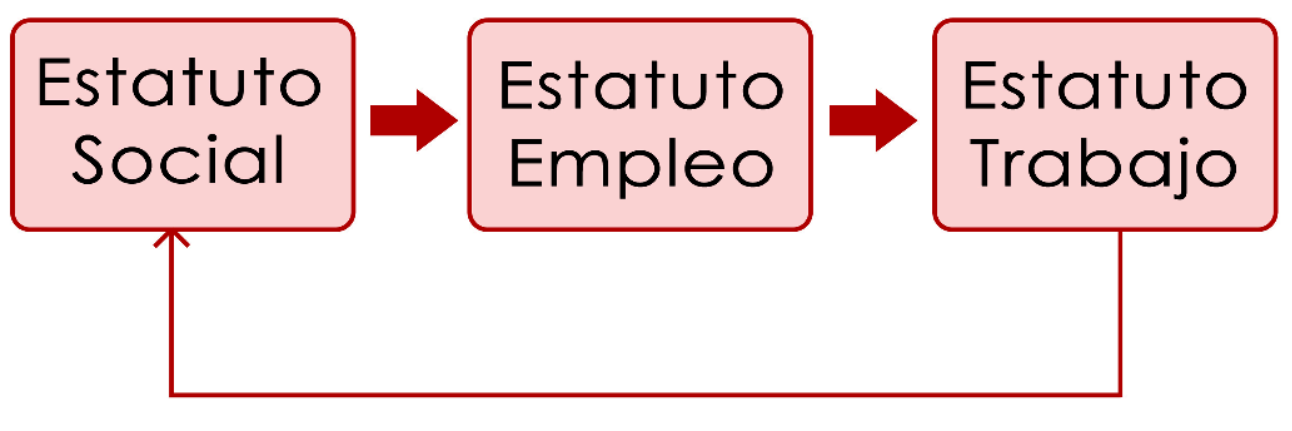

Figura 1. Jerarquización del empleo y el trabajo en el orden social. Elaboración propia.

6 En relación a lo anterior, cabría pensar que el/la trabajador/a, al diferenciar empleo y trabajo, podría rechazar uno y estar comprometido con el otro. Así, puede resistirse al y sus condiciones, pero tener apego por el empleo y desear mantenerlo, pues muchos de 
los elementos que son considerados como parte del trabajo, como son la cualificación, jornada laboral, salario, entre otros, en gran medida, dependen del estatus del empleo.

7 Estas relaciones contradictorias entre trabajo y empleo, afectan tanto a hombres como a mujeres; sin embargo, en el caso de estas últimas, dicha contradicción sería más patente. Por un lado, el trabajo para ellas se sitúa entre los ideales de autonomía y necesidad de subsistencia (Maruani, 2000), y por otro, el acceso a empleos de mayor flexibilidad temporal, con el fin de conciliar el trabajo doméstico y el trabajo remunerado, lo que en muchas ocasiones trae consigo precarización, vulnerabilidad social y laboral. En este sentido, puede plantearse que las mujeres son las más presentes en empleos de bajo estatus, lo que muchas veces se traduce en desregulación de la jornada laboral, falta de cotizaciones previsionales, bajos salarios, entre otros. Esto se relaciona con un reparto sexual del empleo, en que mujeres y hombres pueden realizar un mismo trabajo, pero con un estatus diferenciado, y al mismo tiempo es posible evidenciar la división sexual del trabajo, en lo que respecta a las actividades laborales que hombres y mujeres efectúan, ya que aun cuando ocupen un mismo cargo laboral, las tareas que realizan pueden ser distintas en cada caso.

\subsection{Flexibilidad de la jornada laboral femenina}

La experiencia internacional, ha evidenciado que las mujeres eligen el tiempo parcial de trabajo remunerado, dado que éste permitiría conciliar, de mejor forma, la esfera familiar y laboral (PNUD, 2010). Para contextualizar la situación en Chile, es importante señalar que el $67,8 \%$ de las personas ocupadas, corresponden a hombres, mientras que el 43,5 \% a mujeres (Instituto Nacional de Estadísticas, 2011). De estas últimas, un $24,4 \%$ se desempeñan en empleos de tiempo parcial (Rau, 2011), tasa similar a la de los países de la OCDE, pero baja en comparación con aquellos que tienen una mayor participación femenina en el mercado laboral (PNUD, 2010).

9 Por lo anterior, es relevante estudiar el tiempo de trabajo de las mujeres, toda vez que las tensiones entre las responsabilidades domésticas y el trabajo remunerado, es uno de los principales motivos que aducen las éstas para no participar en el mercado laboral (Comunidad Mujer, 2011a ; PNUD, 2010). Como respuesta a esto, se le ha dado un lugar fundamental a la creciente flexibilidad de la jornada laboral, entendida como la adaptación eficiente por parte de las organizaciones al contexto de continuo cambio económico (Yáñez 1999). Cabe destacar, que la jornada ordinaria de trabajo en Chile, consiste en 45 horas semanales, en contraste con la jornada parcial, que no supera los dos tercios de la primera.

Los efectos que esto ha tenido en Chile, no son neutros. El bajo nivel educacional de mujeres de sectores sociales más vulnerables, hace que éstas no encuentren trabajo en empresas del sector formal, o bien que sus empleos tengan jornadas laborales ambiguas y poco reguladas (PNUD, 2010). En la actualidad, y a pesar del avance que ha tenido la inmersión de las mujeres en el mundo del trabajo, la participación laboral femenina es una de las más bajas en comparación con países miembros de la OECD. Así mismo, pese a los mayores niveles de ingreso en relación a los demás países de Sudamérica, la brecha de participación laboral entre hombres y mujeres en Chile es la más alta (Comunidad Mujer, 2011b). Radica en ello la relevancia al estudiar el empleo particularmente en este país y en el resto de Sudamérica, tanto a nivel académico y profesional como en la consecuente creación de políticas públicas (Figura 2). 
Figura 2 - Brecha de la Participación Laboral entre Hombres y Mujeres en año 2009. Comunidad Mujer (2011b)

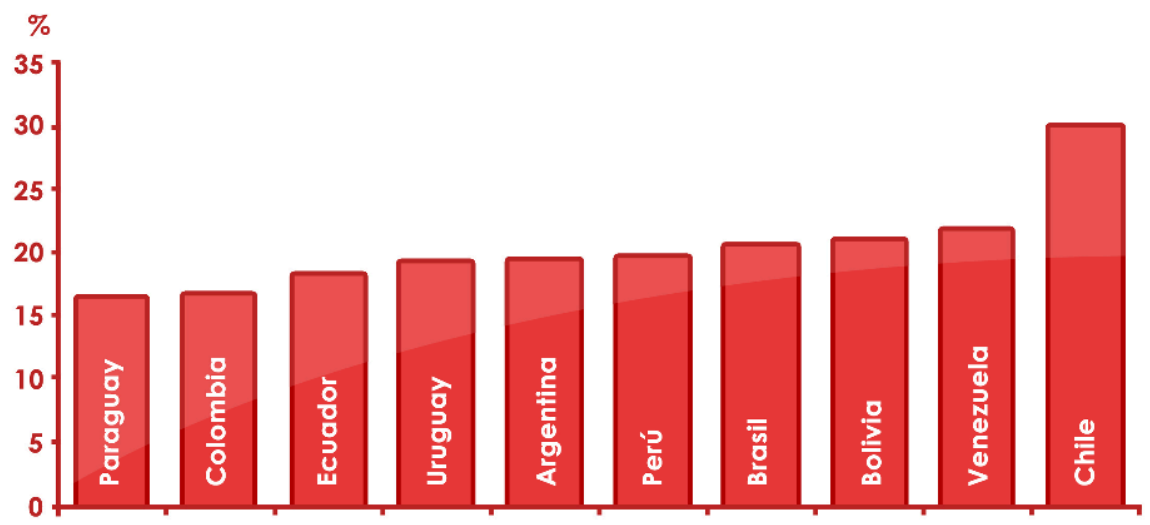

Figura 2: Brecha de la Participación Laboral entre Hombres y Mujeres en año 2009. Comunidad Mujer(201 lb)

11 Un segundo problema es la doble jornada laboral femenina. Esta se refiere a la suma de tiempos de trabajo, tanto del ámbito remunerado, como del doméstico, carga que principalmente recae en las mujeres. En tal sentido, según la Encuesta de Caracterización Socioeconómica Nacional (CASEN, 2009), ocho de cada diez mujeres realizan, de lunes a viernes, un promedio de media hora de tareas domésticas -como son : preparación de alimentos, cuidado de personas, administración del hogar, pago de cuentas, entre otras-, en contraste con cuatro de cada diez hombres, en el mismo período (Instituto Nacional de Estadísticas, 2009).

Lo anterior se traduce, en que un 77,2\% de las mujeres dedican, de lunes a viernes, 3,9 horas diarias promedio a trabajo doméstico - lo que se acrecienta los fines de semana a un $81,9 \%$, destinando 4,1 horas diarias- . Mientras que el $31,7 \%$ de los hombres realizan estas labores de lunes a viernes - cifra que aumenta los sábados y domingos, llegando a un 57,3 \%- (Instituto Nacional de Estadísticas, 2009). De este modo, los hombres, tienen una baja participación en el trabajo doméstico, incluso si no están empleados (Comunidad Mujer, 2012).

Respecto a lo anterior, puede desprenderse que el cuidado del hogar se visualiza como una actividad no calificada, que se da naturalmente en las mujeres, repercutiendo directamente en sus condiciones laborales (Comunidad Mujer, 2012). En tal sentido, existe una doble relación entre maternidad y mujer trabajadora. Por un lado, mujeres que son madres ven muchas veces coartadas sus posibilidades de acceder a buenos empleos o el ascenso a ciertos puestos de trabajo. Por otro lado, aquellas mujeres trabajadoras, en ocasiones ven obstaculizadas sus posibilidades de ser madres.

La importancia del estudio de esta temática, es que no sólo nos brinda conocimientos respecto al mundo del trabajo, sino también del funcionamiento de la sociedad. Por esto, la redistribución de los tiempos de trabajo, configuran las relaciones sociales al interior de las organizaciones, al segmentar a los trabajadores/as entre aquellos/as que tienen horarios regulares y los que tienen tiempos imprevisibles y ambiguos. Para Maruani (2007), las variaciones del tiempo de trabajo, poco tienen que ver con el tiempo concreto, sino más bien, dicen relación con una norma social. De esta manera, existiría una relación laboral que presupone la homogeneidad de experiencias de vida y 
proyectos laborales, como normalidad. Así, el trabajo a tiempo parcial, se constituye como una revocación a esta norma social, por lo tanto "no es el número de horas trabajadas el que constituye la unidad de categoría, sino el hecho de que existe una derogación a la norma" (Maruani, 2007, p. 87).

Por lo anteriormente expuesto, el artículo que aquí se presenta aborda algunas de las problemáticas actuales que afectan a las mujeres chilenas de clase media en su integración a las dinámicas laborales, en torno a diferenciación de trabajo y empleo, las consecuencias derivadas de la flexibilización laboral y la doble jornada de trabajo. Por medio de la realización de análisis de discurso en entrevistas realizadas a distintas trabajadoras, se extraerán conclusiones relevantes al momento de comprender el escenario actual en que se encuentra el trabajo femenino en Chile, y aportará elementos que permitan avanzar en el saber teórico y práctico del psicólogo organizacional en la comprensión de este fenómeno, así como en la resolución de las distintas problemáticas que conlleva para la mujer chilena

\section{Metodología}

16 El presente estudio es de carácter cualitativo. En este sentido, se entenderá por métodos cualitativos aquellos procedimientos que buscan principalmente la comprensión de los procesos sociales y dar cuenta de ellos, más que la explicación y predicción de los mismos; basándose en herramientas analíticas que yacen en la interpretación (Iñiguez, 2008). Por otra parte, la temática se abordará desde una perspectiva construccionista. En tal sentido, Burr (1995), señala entre sus características : anti- esencialismo ; lenguaje como forma de acción social; foco en la interacción social; lenguaje como una pre - condición para el pensamiento; especificidad histórica y cultural del conocimiento; anti-realismo y foco en los procesos.

17 Las directrices que guían el estudio son tres : a) el punto central de la investigación son los discursos de mujeres de la Región Metropolitana de Chile; b) dichos discursos se analizarán, utilizando para ello análisis de discurso ; c) el carácter es inductivo.

\subsection{Participantes}

18 Se utilizó un muestreo en bola de nieve, que consiste en la ampliación de los sujetos participantes, partiendo de contactos recomendados por otros informantes claves. De este modo, se trabajó con los discursos de 17 mujeres chilenas, de la Región Metropolitana, de estrato socio-económico medio, con hijos y con un rango etario que fluctúa entre los 25 y 40 años. Todas las participantes tuvieron o tienen en la actualidad empleos de carácter flexible, ya sea por su jornada laboral -a medio tiempo o por turnos y horas- o por el lugar donde se lleva a cabo el trabajo - en el domicilio, en oficinas satélites, etc.- En términos educacionales siete tienen estudios profesionales universitarios y diez no.

19 Al momento de ser entrevistadas, 7 de las participantes trabajaban en una institución pública del Estado de Chile, dependiente del Ministerio de Hacienda. Contaban con educación técnico-profesional (ninguna de ellas era universitaria). Sus cargos correspondían al nivel operario, ubicado en la base jerárquica dentro de la estructura organizacional. Se puede agregar que todas contaban con contrato a honorarios -que 
por definición no establece horarios laborales fijos-, y trabajaban desde su hogar. Aun así, se les exigía cumplir con una jornada laboral de 8 horas diarias, tiempo insuficiente en relación a su carga laboral, por lo que recurrentemente trabajaban en jornadas más largas que la requerida por la institución.

Las restantes 10 entrevistadas, correspondían a mujeres jóvenes que habían recibido educación universitaria completa en profesiones tales como ingeniería civil, antropología, sociología e ingeniería en turismo (en el caso de la asistente de vuelo), todas de estrato socio económico medio.

Cabe señalar que no es fácil definir la clase media en Chile. Es un grupo con una heterogeneidad alta, lo que dificulta una definición única de ella. Este grupo social no sufre los embates de las clases más populares, pero tampoco tienen seguridad en su posición social. Tienen altos niveles de precariedad, inestabilidad y protección social limitada (Espinoza \& Barozet, 2008). En este sentido, un aspecto relevante a considerar a la hora de definir la clase media en Chile, es aquel que refiere a las condiciones laborales. Es un grupo asalariado, en que a diferencia de sectores populares, el trabajo tiene un carácter de regular y permanente en la vida cotidiana de los/as sujetos (Espinoza \& Barozet, 2008). En términos de ingreso familiar mensual, la clase media es constituida por los grupos C2 y C3, los que perciben entre $\$ 550.000$ (1.091 US) y $\$ 1.500 .000$ (2.976 US) (Méndez, 2008).

Estas participantes se desempeñaban en el área privada, contando dos de ellas con contrato de trabajo a plazo fijo, y las restantes con convenio de honorarios. Cuatro de las diez participantes trabajaban a tiempo parcial, mientras que las seis restantes lo hacían por horas o turnos. A su vez, estas profesionales eran empleadoras de mujeres asesoras del hogar, sin embargo, sólo en algunos casos la relación laboral estaba mediada por un contrato de trabajo indefinido o a plazo fijo y en los otros casos tal contrato no existía, sino mas bien se daba por acuerdos de palabra.

A partir de las entrevistas realizadas, se recogió información diversa en torno a las experiencias laborales de las trabajadoras y un acercamiento a distintas posiciones sobre las condiciones flexibles en las que se encontraban ejerciendo sus funciones, a partir de lo cual fue posible generar un panorama actual acerca de las condiciones laborales y la relación establecida entre empleo y trabajo en la región.

En relación a la muestra, cabe señalar que no se busca la representatividad en términos estadísticos, como se utiliza en investigaciones cuantitativas. La representatividad está dada por el hecho de que la informante está "actuando" como si estuviera en un rol, donde lo que interesa no son las características individuales de ésta, sino que es parte de un grupo social particular. De esta forma, las participantes provienen de una posición social específica, lo que hace que sus discursos no sean construidos puramente desde sus individualidades, sino que cada relato da cuenta de una posición de sujeto social (Sisto \& Fardella 2009).

\subsection{Acercamiento teórico del análisis}

Se utilizó análisis de discurso, para el procesamiento de la información. En torno a éste, hay variadas definiciones, dependiendo de la tradición desde la cual se erigen : la teoría de los actos de habla, la etnometodología de la comunicación, la sociolingüística interaccional, la pragmática, etc. (Iñiguez, 2003). Para los efectos de este estudio, se entiende Análisis de Discurso como: "El discurso es un conjunto de prácticas 
lingüísticas que mantienen y promueven ciertas relaciones sociales. El análisis consiste en estudiar cómo estas prácticas actúan en el presente manteniendo y promoviendo estas relaciones" (Iñiguez, 2003, p. 99). Este tipo de análisis es especialmente relevante en este estudio ya que permite visibilizar ciertas prácticas que habitualmente son invisibilizadas socialmente. En tal sentido, este método problematiza aquello que ha sido instalado como obvio, más aún, en lo que respecta al ámbito de los estudios de género y trabajo, posibilita la desnaturalización de las relaciones de poder que "sostienen o perpetúan las inequidades que nos interesan" (Aguayo, 2009, p. 3).

A su vez, en estudios respecto a temáticas relacionadas con el mundo del trabajo, es especialmente relevante por variadas razones. En una primera instancia, permite comprender el trabajo como un lugar de construcción de significados, discursos y subjetividades, lo que conlleva ir más allá de las miradas de los/las y los trabajadores/ as como recurso a ser gestionado, quedando la importancia, exclusivamente en el ámbito de producción económica (Stecher, 2009). De esta manera, es posible visibilizar la heterogeneidad de posiciones sociales - mujeres, hombres, indígenas, etc.significados y producciones discursivas, al interior del mundo laboral. Así se da cuenta de vivencias, trayectorias vitales y laborales altamente desiguales y muchas veces, excluyentes.

Lo anterior se enmarca en contextos socio-históricos. En lo que refiere a las últimas décadas, el desarrollo de lógicas de producción y acumulación capitalista, de carácter global y flexible ha generado reestructuraciones progresivas en la esfera del trabajo, habiendo articulaciones entre transformaciones sociales, políticas y cambios de carácter laboral. De esta forma, se puede dar un acercamiento mayor a las transformaciones y formas que adquieren los procesos socio-productivos en América Latina, en que la flexibilidad laboral ha significado, principalmente la precarización de los empleos y la desregulación de los mercados laborales (De la Garza, 2002).

Por último, cabe señalar que los cambios producidos en el mundo del trabajo están asociados a transformaciones y rearticulaciones a nivel discursivo. Esto ha significado la articulación del discurso sobre imaginarios respecto al trabajo, las organizaciones y la economía capitalista; nuevas interacciones entre jefaturas, trabajadores/as y autoridad; nuevas construcciones discursivas en relación a posiciones subjetivas.

\subsection{Procedimiento}

Como instrumento de investigación se utilizó la entrevista abierta semi directiva, en la cual las preguntas están dirigidas hacia ciertos temas relevantes a tocar (Valles, 1999), contemplando tres temáticas centrales : 1) información sobre condiciones laborales y el discurso relacionado con el empleo y trabajo 2) conocimiento sobre la organización del trabajo doméstico 3) discursos asociados a la maternidad y trabajo. Cabe destacar, que se resguardó la confidencialidad y anonimato de todas las participantes de la investigación.

Los resultados fueron sometidos a criterios de validez para datos cualitativos. Se generó un proceso de triangulación, en el cual éstos fueron presentados a las participantes. Todas manifestaron conformidad con la interpretación de los datos obtenidos, por lo tanto, es posible afirmar que éstos poseen la fidelidad y veracidad necesaria para los fines de este trabajo. En este sentido, Bonilla-Castro y Rodríguez (2005), sostienen que la validez interna estará dada, por una parte, por la credibilidad de los resultados, y por 
otra, por la coherencia de estos con la investigación. De la misma manera, la validez externa se provee, en tanto los resultados obtenidos permitan ser aplicados a grupos similares, principalmente, porque no se funda en criterios estadísticos, sino en la posición social de los/as sujetos.

Como forma de organizar y dar validez interna a los resultados, se procedió de la siguiente manera :

- Transcripción de las sesiones de entrevistas: A partir del audio, las grabaciones se transcribieron, pasando de lo oral a lo escrito. Dicha transcripción hace posible una familiarización con el material y una primera impresión global. Se consideró para producir un registro que capturara la mayor parte de la situación dada en la entrevista, a una persona como facilitadora del proceso, quien realizaría la entrevista y otra que observaría.

- Identificación de los principales ejes argumentativos de los discursos: Primeramente, se preparó el material y se analizó el cuerpo discursivo en su totalidad de acuerdo a las tres temáticas centrales. En una segunda etapa, se realizaron lecturas sucesivas, aunque no siempre siguiendo el orden formal del texto, a fin de deconstruir los datos. Además se tomaron notas y se destacaron extractos a utilizar en el análisis, siempre con la consideración que estos fueran contenidos literales entregados por las entrevistadas.

- Análisis de elementos discursivos: El análisis se divide en dos partes: estructura de la narración y estrategias discursivas. De esta manera, se tomó en consideración el contenido y forma del discurso.

\section{Resultados}

El análisis aquí presentado, surge a la luz de la selección de las citas más relevantes de acuerdo a las problemáticas planteadas en este artículo, que dan cuenta del discurso general de las participantes. Cabe destacar, que al momento de generar las entrevistas y seleccionar las citas, no se contaba con ideas preconcebidas acerca de los posibles resultados a obtener.

El grupo de mujeres con que se trabajó posee características sociales específicas, descritas anteriormente, correspondientes a mujeres residentes en la región Metropolitana de Chile, de clase media y con trabajos de carácter flexible. De ninguna manera se pretende con los resultados generalizar o extrapolar sus conclusiones a otros grupos de mujeres, más bien, se busca dar ciertas claves de lectura al momento de analizar conjuntos sociales de características similares- a modo de validez externa-, tomando en consideración los límites propios de todo estudio.

Del análisis de las entrevistas obtenidas, es posible desprender tres ejes discursivos sobre la jornada laboral sexuada, que apuntan a la relación y de trabajo y empleo, la flexibilización del trabajo femenino y la legitimación de la doble jornada laboral.

\subsection{Estatus, trabajo y empleo : riesgos y consecuencias para las trabajadoras}

35 Aunque las nociones de trabajo y empleo tienden a ser equivalentes, ante la pregunta por la diferencia entre un término y otro, aparecen algunas divergencias. 
(El trabajo) Es una forma de subsistir, porque tranquilidad no me da. Ni me puedo proyectar ni nada, es una forma de sobrevivir (Aída, operaria, 35 años). pesar de lo exiguo que resulta su producto. Lo que permite la subsistencia es la remuneración recibida por el trabajo. Las condiciones de trabajo cotidianas no le generan tranquilidad, y el contexto del mismo no permite una proyección laboral o personal. Todos estos elementos son parte de las condiciones que enmarcan la actividad productiva, vale decir, el empleo.

En la Universidad, teníamos dos auxiliares : uno vivía en La Pintana y el otro por azares del destino vivía en Vitacura. Claramente al que vivía en Vitacura lo trataban mucho mejor, lo mandaban hacer cosas más livianas que al gallo que vivía en La Pintana. Si había que mover una caja de 50 kilos, no iba el chico de Vitacura que era más joven; no, iba este caballero que vivía en La Pintana y que tenía 10 años más (Mirta, administradora de productos, 30 años).

En este relato se aprecia cómo el estatus social al que pertenece el trabajador influye en el estatus de su empleo, y ello a su vez en el valor que adquiere el trabajo. A pesar de que ambos trabajadores realizaran la misma labor, existían distinciones en las condiciones de su actividad productiva, es decir, en las características y estatus del empleo, debido únicamente a la supuesta condición social que otorga el hecho de vivir en un lugar determinado. Ello genera inmediatamente, y en concordancia con éste, un cierto estatus y valoración del trabajo realizado, y al mismo tiempo, diferencias en sus condiciones de trabajo en perjuicio del trabajador que habita en el lugar de baja categoría social, siendo afectada negativamente su calidad de vida laboral.

Estar aquí es como estar en la cúspide del Everest, si tú salís al mundo con la imagen de que trabajái acá soy toda poderosa [...] El estar acá es muy valorado, yo me he dado cuenta por los pitutos que he hecho... nosotros llegamos como pollitos, ahora somos capaces de desenvolvernos en cualquier parte. Prácticamente te prenden una vela...(Sandra, operaria, 38 años).

En este relato, es posible apreciar cómo se construye un modelo de legitimación y validación del trabajo frente al "mundo", vale decir, ante otras instituciones y personas externas al lugar de trabajo. La imagen positiva que proyecta el lugar donde se desempeñan laboralmente, da status al empleo que poseen las trabajadoras y las inviste de poder.

De esta manera, el empleo adquiere una nueva connotación, puesto que entrega más que la posibilidad de trabajar : el conocimiento adquirido y el status del empleo crea confianza y seguridad en sí mismas, por la visión que, de ese empleo, se tiene en el ámbito relacionado. Otorga refuerzo personal, ayuda a levantar la autoestima y permite situarse ante el mercado laboral y en otros rubros en una situación de ventaja.

Para mayor abundamiento, el aprendizaje también significa desarrollo y cambios personales. De lo que aparece en el discurso, se desprende que en la especificidad del aspecto productivo del trabajo que se realiza, existe un aprendizaje que posibilita construirse como ser humano en todas sus dimensiones. Asimismo, como se ha dicho, claramente se manifiesta el desarrollo personal como proceso a través del trabajo. Las

Laboreal, Volume $8 \mathrm{~N}^{\circ} 1$ | 2012 
posibilidades que ofrece el trabajo que se realiza, son reconocidas en su dimensión positiva sólo a partir del carácter que se le confiere al empleo y a la organización en que se trabaja.

Es súper injusto lo que nos pasa, porque nosotros somos los que tenemos parada esta cosa, acá se transan millones de dólares y manejamos información que es muy importante... yo no sé en qué nivel nos ven a nosotros [...] Nuestro empleo es muy mal calificado... es muy injusto (Sandra, operaria, 38 años)

En este caso, la misma entrevistada se posiciona a sí misma como perteneciente a un grupo de personas que, a pesar de realizar un trabajo muy importante para la organización que dice relación tanto con la envergadura del capital que se mueve como con la información que se maneja, su nivel de valoración por parte del organismo es menor al que creen merecer.

De esta manera, aun cuando la entrevistada comenta que la organización se mantiene en pie gracias a las labores que ellas realizan, no hay un reconocimiento adecuado de su desempeño, lo cual genera una percepción de incertidumbre ante la valoración y lugar de trabajo, acompañado de la certeza de la baja calificación del mismo.

El bajo status del empleo precariza la labor de las trabajadoras y sus condiciones de trabajo se vuelven desfavorables en el ámbito cotidiano de manera encubierta, a través de la inestabilidad y fragilidad que adquiere la situación laboral. Se produce así desvalorización de las mujeres trabajadoras y pocas expectativas de desarrollo y proyección laboral dentro de la empresa.

\footnotetext{
Hay una discriminación muy fuerte por la manera con que la gente se ve, me tocó trabajar con unas que no coincidían con la imagen ni de inmigrante aimara o no tenían muy marcado lo indígena, esta típica etiqueta de "cholo" [...] en esta sociedad discriminan por cuna, tanto en el punto de vista de la ubicación geográfica como en la étnica, sus perspectivas laborales están restringidas (Pamela, socióloga, 32 años).
}

De la cita anterior se desprende que la valoración de la actividad productiva de las trabajadoras está fuertemente mediada por la percepción que de ellas tienen sus empleadores, en cuanto a su nivel socioeconómico, lugar de origen y aspecto físico. De esta manera, la condición de inmigrante de las empleadas y el status social en el que se ubican, inciden en las diferentes situaciones de discriminación de las que son parte, y actúan como condicionadores del estatus del empleo al cual podrían acceder, influyendo negativamente también en las oportunidades laborales y condiciones de trabajo posibles de adquirir estando en un país extranjero.

\subsection{Flexibilidad Laboral : entre el trabajo remunerado y la precariedad}

Al ingresar al mercado laboral, las mujeres han visto alterada su jornada de trabajo, generando un aumento de la misma en cuanto a tiempo y funciones, producto del trabajo realizado dentro y fuera del hogar, lo que provoca sensaciones de cansancio y pérdida de libertades, entre otras cosas.

Acá el trabajo es tan pesado... yo era un zombie, a puro café y más encima tenía que mamarme las cosas de la casa... yo misma me di cuenta que tenía 
que hacerme el tiempo para mí, yo no puedo con todo... [...] Me gustaría estar en una sola pega, llegar a la casa y desenchufarme, en la medida en que pasan las horas, como que mi reloj va diciéndome lo que tengo que hacer, como que estoy programada... (Sandra, operaria, 38 años)

46 Se aprecia en este relato cómo la flexibilidad laboral en el trabajo remunerado, que supuestamente actúa en pos de una integración y nivelación entre la labor doméstica y la productiva externa al hogar para la mujer, actúa directamente en perjuicio de las condiciones laborales; lo cual produce una ampliación de la jornada laboral que absorbe gran parte del tiempo personal, generándose una rutina inalterable en la realización de ambos tipos de labores y creándose así la percepción de falta en el tiempo dedicado a sí misma, agotamiento y desmedro en su desempeño. Ello, además, influye en la posibilidad de descansar apropiadamente y del disfrute del tiempo libre para otros tipos de actividades de desarrollo personal. De esta manera, las mujeres no cuentan con la posibilidad de organizar sus tiempos de forma adecuada, viviendo en la constante presión de responder a ambos tipos de labores.

Sumado a lo anterior, y a pesar de provocar esta problemática en la vida femenina, la realización del trabajo no remunerado permanece invisibilizado, ubicando a las mujeres en una situación de desprotección continua, en la que las funciones domésticas terminan por generar un agotamiento en la vida diaria.

En las tres entrevistas en que fui y me dijeron que no... porque tenían que mandarme a la casa en móvil cuando salía muy tarde. Yo dije : "no... aquí yo me equivoqué de carrera, no debí haber estudiado esto, yo debí haber estudiado para secretaria con horarios limitados y listo (Aída, operaria, 35 años).

En la cita se aprecia la justificación utilizada para discriminar a las mujeres en el mundo laboral, relacionada con las aparentes dificultades que presenta el horario de trabajo nocturno, respecto a la movilización especial que requieren las trabajadoras para trasladarse a su lugar de residencia. Esto, debido a la vulnerabilidad y debilidad que a ellas se asocia, obviando el hecho de que un hombre está sujeto a los mismos riesgos al trabajar por la noche. Esta discriminación termina por generar inestabilidad en la concepción del empleo y tal incertidumbre para la trabajadora, que la lleva a cuestionar incluso su elección profesional.

Es como un trato implícito que tenemos, no está nada escrito pero yo sé que si yo le pido "Rosita quédate un rato más" ella se queda... llega a las 9 y se va a las 6 , ehm, no sé cuántas horas son, pero creo que es como lo legal. Y bueno, ella tiene la libertad de almorzar y tomarse el tiempo que quiera, de tomar once, de parar para descansar. En ese sentido yo soy súper relajada, ni siquiera cuando estaba puertas adentro me importaba el tiempo que se tomara en hacer las cosas de la casa. (Tatiana, asistente de vuelo, 34 años).

De este relato se desprende que las condiciones de la trabajadora se caracterizan por ser poco claras e informales, y nacen a través de acuerdos mutuos entre la mujer empleada y el empleador correspondiente, sin contrato de por medio. La empleadora incluso, no tiene conocimiento preciso sobre los aspectos legales que se requieren para contratar a alguien, y tampoco la empleada cuenta con un respaldo legal que la proteja. Así mismo, no existe claridad en las condiciones del trabajo, como son los horarios, los tiempos de alimentación y de descanso. 
50 De esta manera, se aprecia cómo la flexibilización laboral refuerza y legitima un sistema desigual, sin permitir a la mujer integrar las distintas áreas de su vida, y coartando al mismo tiempo la posibilidad de acceso a ciertos empleos.

51 Al manifestarse la flexibilización en oferta de empleos a tiempo parcial y en condiciones poco definidas, se genera precariedad en las condiciones de trabajo, en términos de estabilidad laboral, regulación legal y protecciones sociales, lo cual disminuye sus posibilidades de desarrollo económico y profesional.

52 Los efectos a nivel social que tiene la flexibilización laboral para las mujeres, se relacionan con la marginación y exclusión de la que son parte a nivel tanto profesional como económico, teniendo que enfrentarse a un sinnúmero dificultades a la hora de integrase al trabajo, que para muchas, éste representa un sistema primordial que influye en sus aspiraciones y posibilidades de proyección, tanto a nivel profesional como personal.

53 Se produce entonces un perjuicio en el desarrollo laboral y también en la vida privada, pues las condiciones de empleo no son adecuadas a la hora de compatibilizar el desarrollo laboral con el área familiar.

54 Esto demuestra cómo la flexibilización laboral desprotege socialmente y desvaloriza a las mujeres, perjudicando sus condiciones laborales y arriesgándolas a potenciales situaciones de explotación laboral y riesgo en la mantención y ejercicio de sus derechos como trabajadoras. Junto con esto, la inexistencia de contrato en algunos casos, demuestra la informalidad en cuanto a las normas de regulación y la falta de rigor en el trato hacia las empleadas, lo cual supone una relación laboral desigual, abusiva e injusta.

En síntesis, se encuentran factores al exterior de la organización -como el reconocimiento del trabajo y pertenencia a una organización destacada dentro del rubro-y también al interior de ésta -por ejemplo, la valorización por parte del empleador de las labores realizadas y condiciones contractuales de las mujeres- que ejercen influencia en el estatus del trabajo y por lo tanto del empleo, siendo aspectos claves a considerar en cuanto al reconocimiento y valoración sociales hacia las mismas en el trabajo remunerado. A su vez, el modelo de flexibilización de las condiciones laborales vigentes en la actualidad, propicia las situaciones de precarización laboral, produciendo ambigüedades en las condiciones de trabajo, debiendo las empleadas complementar el trabajo remunerado con el de otras áreas como la familiar (Figura 3). 
Figura 3 - Flexibilidad y reconocimiento social del trabajo y del empleo de las mujeres. Elaboración propria

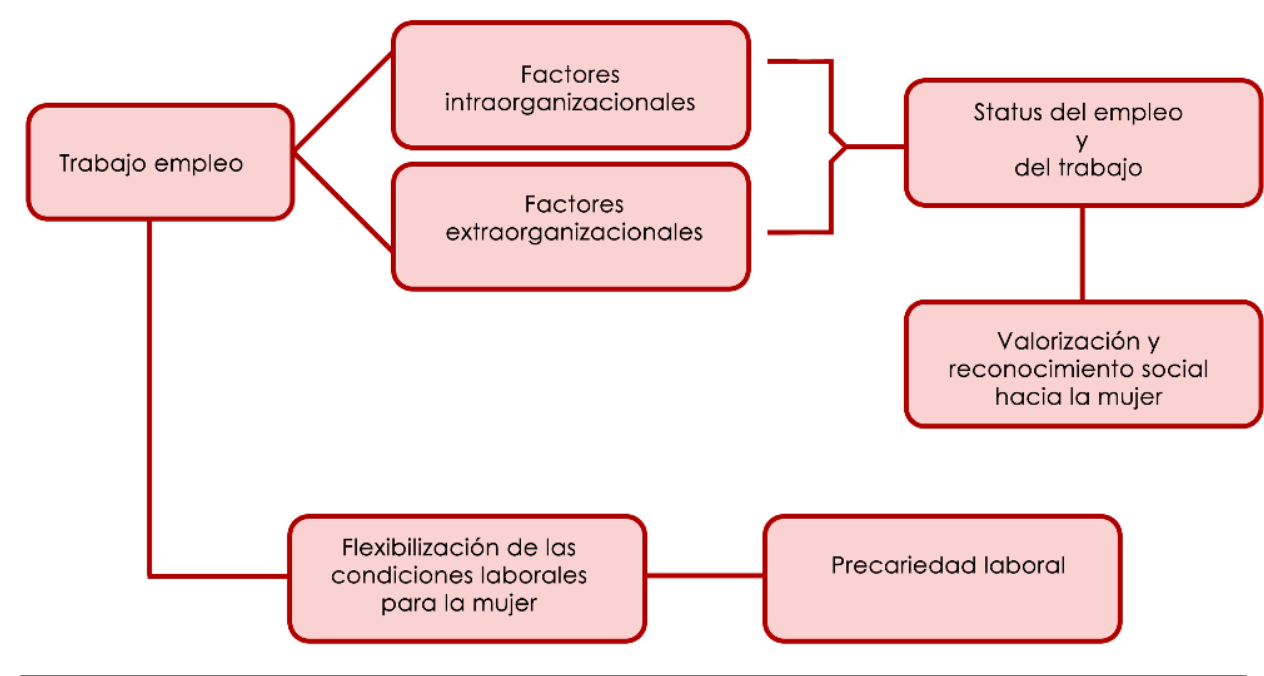

Figura 3. Flexibilidad y reconocimiento social del trabajo y del empleo de las mujeres.Elaboración propia.

\subsection{Maternidad : comienzo y final de la doble jornada laboral femenina}

El movimiento de las mujeres hacia el mercado laboral, no ha sido un camino expedito, fácil ni vacío de discriminaciones. El mismo hecho de ser mujer -con o sin hijos-, en ocasiones se transforma en un problema a la hora de encontrar trabajo.

Uno me dijo una vez: no, después les da por tener hijos y hay que andar dándoles licencia. No hay por dónde... si no es por lo que estudié, es porque soy mujer (Aída, operaria, 35 años).

$Y$ es que un gran argumento para no contratar a mujeres en edad fértil es que la mujer se embaraza, teniendo que abandonar el trabajo por un tiempo mediante licencias pre y posnatal. La aparente dificultad que se desprende de ello es la pérdida de productividad en el trabajo debido a su ausencia, generando potenciales bajas en los productos y ganancias de la organización.

Con respecto a su tránsito desde ámbito privado al público, vale decir, del trabajo doméstico al trabajo productivo externo, las mujeres han debido transformar sus pautas de acción para sopesar ambos tipos de labores. Esto no significa que uno haya sido reemplazado por el otro. En la mayoría de los casos, las mujeres deben lidiar con dos jornadas laborales, aquella que se realiza dentro y fuera del hogar.

En este contexto, las mujeres, sobre todo cuando son madres, sienten que no puede realizar ambos trabajos a la vez, por lo que se ven necesitadas de un apoyo en las labores cotidianas, y en ocasiones contratan a otra mujer que le ayude a lidiar con las tareas del hogar. La empleada doméstica o "nana", entonces, es vista como un soporte en el trabajo del hogar, realizando labores relacionadas con la limpieza de la casa, cocina y cuidado de los hijos. 
Yo siempre consideraba que si los dos trabajábamos, mínimo tenía que haber alguien que hiciera las cosas de la casa, porque tú no puedes trabajar afuera y en la casa también, por esa razón tengo nana, por tener a alguien que ayudara con las cosas de la casa (Cristina, Ingeniera, 40 años).

Tengo nana porque trabajo y los niños cuando llegan del colegio, alguien los tiene que recibir, darles la comida y los tiene que cuidar cuando yo no estoy (Pamela, socióloga, 32 años).

60 más que por la incompatibilidad fáctica de ambas labores, por una sobrecarga laboral que se desea evitar. La ayuda en las tareas del hogar viene a ser un respaldo concreto que permite a la mujer realizar el trabajo productivo y mantener cubiertas las necesidades del hogar. Así, el reemplazo de una mujer por otra viene a desplazar la preocupación existente por el trabajo doméstico, en relación tanto a las labores hogareñas como a las relacionadas con el cuidado de los niños.

61 Al respecto, a la hora de aliviar la doble jornada laboral, la labor del cuidado de los hijos supone el eje fundamental del servicio contratado, ubicándose en una prioridad más alta que el cuidado del hogar en general. Por esta razón, algunas mujeres, mientras consideren que el cuidado de sus hijos está resuelto, se sienten satisfechas y realizan por sí mismas el trabajo doméstico que queda pendiente.

Yo tuve mi primera nana, era súper viejita la nana, pero cuidaba súper bien a la Sofía y al Joaquín también, así que al final igual yo terminaba haciendo, entre que yo trabajaba, estudiaba, terminaba el fin de semana encerando, limpiando los vidrios, pero eso ya no importaba porque me cuidaba bien a la guagua. (Alejandra, ingeniera, 37 años).

62

De esta manera, la preocupación fundamental a la hora de contratar un servicio doméstico es el buen cuidado de los hijos, debido a lo cual, en variadas ocasiones la función de trabajadora queda sujeta únicamente a esta labor, y si bien la empleadora que tiene niños pequeños ya no debe necesariamente permanecer en su casa, continúa realizando una doble jornada laboral al momento de volver a su hogar o los días en que permanece en casa.

Por otro lado, culturalmente el hombre es considerado un sujeto ajeno a lo que tenga que ver con trabajo remunerado femenino, maternidad y doble jornada laboral, limitándose al trabajo remunerado externo al hogar, y como símbolo de proveedor, no realiza labores domésticas. Las mujeres entrevistadas explican la falta de participación del hombre en las labores del hogar, y en cuidado de los niños.

Esto es súper machista, yo lo sé, pero nos criaron así [...] la lógica de la atención de cuidar al otro está instalada como un microchip desde el minuto en que terminas de decir tu primera palabra (Andrea, socióloga, 29 años).

64

En este relato es posible apreciar que, si bien la justificación del rol de la mujer tiene relación con una consideración cultural patriarcal, es difícil abstraerse de ella debido a que se encuentra asentada desde la niñez profundamente. Al mismo tiempo, y por las mismas razones, se justifica el hecho de que el hombre se encuentre ajeno al rol de la mujer en el hogar, naturalizando su papel de trabajador externo al hogar. Esta no es la única manera de explicar por qué el trabajo doméstico es realizado mayormente por mujeres :

Laboreal, Volume $8 \mathrm{~N}^{\circ} 1$ | 2012 
Yo creo que parte porque los hombres no saben hacer las cosas, no tienen dentro de su formación biológica cerebral las tareas domésticas. Muy pocos, quizá no sé, cinco por ciento les nace ordenar su casa o hacer aseo en su espacio pero el resto no sabe o no les interesa (Sofía, antropóloga, 27 años). res domésticas. En ambas es posible apreciar la noción y justificación del rol conferido al hombre como sujeto proveedor y trabajador externo al hogar. Esta justificación viene a actuar entonces como el soporte cultural del trabajo no remunerado realizado por una figura femenina, que sitúa a la mujer como responsable de esta labor antes que el hombre. Y de esta manera, lo que se aprecia en los diferentes discursos al explicar las causas de este fenómeno, constituye la legitimación del mismo, toda vez que las mujeres avalan el hecho de no poseer las mismas funciones que el sexo opuesto mediante razones verídicas y confiables tanto para ellas como para el sentido común. Se observa entonces cómo las mujeres asumen y aprueban el rol que se les ha sido conferido en el hogar, lejos de cuestionarlo y actuar en pos de un repartimiento homogéneo de estas labores, lo que la mayoría de las veces conlleva a una doble jornada laboral en la que el trabajo remunerado productivo se suma irremediablemente al trabajo no remunerado.

En síntesis, existe un adentramiento de las mujeres al trabajo remunerado, paralelo a lo cual no se genera aumento de participación de los hombres en el trabajo doméstico, por el contrario, estos últimos siguen ligados al ámbito productivo externo. Se produce entonces, una sobrecarga laboral hacia las mujeres, encargadas de realizar ambos tipos de labores, por lo que, en algunas ocasiones, contratan a otras mujeres que actúen como soporte en el trabajo privado, concerniente al cuidado de los hijos y demás labores domésticas. La explicación que se realiza en torno al género de la persona contratada y la justificación de la baja participación del hombre en las tareas del hogar -relacionadas con la naturaleza maternal o condición femenina, y a la incapacidad cultural o biológica del hombre para realizar dichas labores-, demuestran la aceptación acrítica de ambos roles por parte de las mujeres y la ausencia del cuestionamiento de las relaciones laborales de género, legitimando así la correspondencia de dichas funciones e impidiendo una transformación destinada a equiparar ambos tipos de jornada laboral. Es por ello que se genera la continuidad y permanencia de la doble jornada laboral femenina (Figura 4). 
Figura 4 - Doble jornada laboral feminina. Elaboración Propria

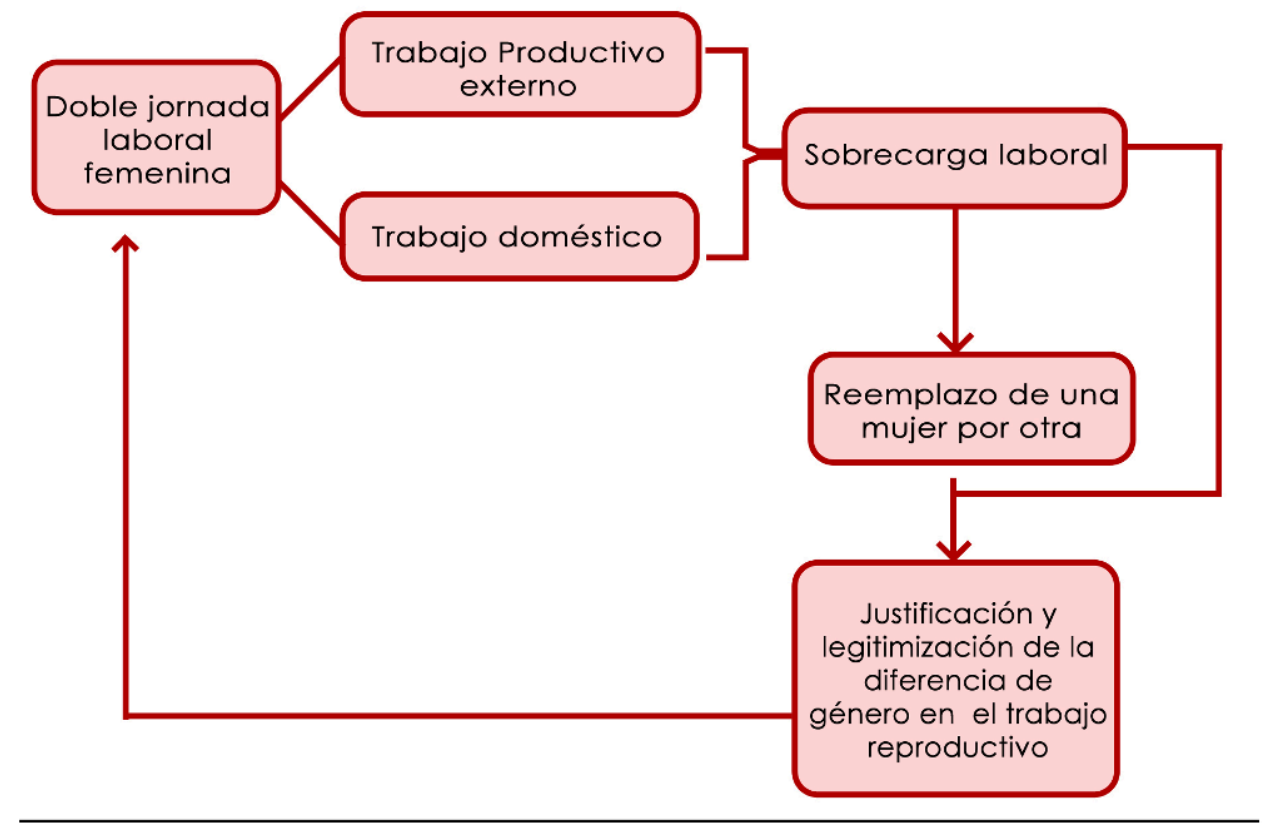

Figura 4. Doble jornada laboral femenina.

Elaboración Propia.

\section{Conclusiones}

El trabajo presentado intenta ser un aporte en cuanto a la investigación sobre jornada laboral femenina en su diferencia con la masculina, en relación al trabajo y el empleo. Se vuelve relevante estudiar este tema, dada las distintas tensiones que se producen al momento de entrar la mujer al mercado del trabajo, teniendo que desempeñarse tanto a nivel familiar como laboral, entre otros ámbitos.

La presentación de las citas expuestas forma parte del discurso de trabajadoras de distintos rubros, que entregan información relevante respecto a la temática mencionada. Aspectos que se abordan en este terreno complejo para la mujer son; empleo y el trabajo, la flexibilización de la jornada laboral femenina, y la maternidad. Independientemente de la actividad desarrollada, se desprenden diferencias discursivas entre los conceptos de trabajo y empleo, ya que aun cuando el trabajo permite la subsistencia a través de la remuneración recibida, éste se ve condicionado por el estatus social al que pertenece la trabajadora pudiendo integrarse o no en distintos rubros. De esta manera, aún cuando los términos de trabajo y empleo son utilizados como sinónimos, es posible que estos presenten diferencias al estudiarlos en mayor profundidad.

71 Tal como lo plantea Prieto (2000), el reconocimiento social del trabajo se produce en tanto empleo, y debido a ello la valorización del trabajo realizado se genera luego de la clasificación social del trabajador/a que lo ejerce. En los relatos analizados se aprecia cómo el estatus social al que pertenece la trabajadora afecta el estatus de su empleo manifestado en sus condiciones laborales, lo cual genera una cierta valoración del trabajo realizado. 

variación en el estatus del empleo ejercido. Ejemplo de ello es el estatus concedido al empleo y el poder que adquiere la trabajadora según la imagen externa con la que cuenta determinada organización, generando una situación ventajosa de la empleada en otros rubros laborales, e incluso efectos positivos en su autoestima, debido al autoposicionamiento como integrante de una organización destacada y relevante. Por otra parte, factores al interior de la organización, como la valoración que el/la empleador/a realiza de las labores desempeñadas por la trabajadora, el respaldo en su desempeño, como también las condiciones contractuales en las que se desenvuelve la relación laboral, podrían llegar a precarizar el estatus del empleo y el escenario para las trabajadoras chilenas. empleo en el desarrollo personal, debido a la necesidad de contar con estabilidad económica y autonomía. Por lo tanto, ello no siempre se vincula con un apego a la organización de la cual se es parte. implementando un modelo de empleo cuyos efectos tienen relación con la precarización, dada las condiciones ambiguas en que se mantiene la relación laboral, en que no se cuenta con la debida regulación y rigurosidad en el ámbito legal y contractual. Yáñez (1999) complementa este punto, indicando que la flexibilidad laboral opera sobre el tiempo de trabajo, intensificando la jornada laboral, lo cual viene a repercutir y afectar directamente en vida de las mujeres, generándose, entre otras cosas, la ambigüedad y/o ausencia del tiempo distribuido para uso personal y recreacional.

Se dificulta también la posibilidad de proyectarse laboralmente, toda vez que las mujeres se ubican en un espacio confuso en términos de condiciones de trabajo, que muchas veces las obliga a mantenerse en la informalidad laboral, lidiando incluso con la discriminación en el proceso de selección. Ello de cuenta de la marginalidad y bajo status que per se tienen las condiciones laborales de la mujer, situación que aumenta sobre todo en clases sociales medias y bajas.

76 Esto, sumado al trabajo doméstico, conlleva una doble jornada laboral manifestada en una constante presión por responder a ambas responsabilidades, lo cual consecuentemente, genera un agotamiento constante y desmedro en la calidad de vida de la mujer. Es por ello que las mujeres han tenido que barajar los tiempos destinados al ejercicio de la maternidad, al trabajo productivo externo y a sí mismas, viendo mermado en algunas ocasiones este último, y necesitando por ello un apoyo externo en la labor doméstica y el cuidado de los hijos, lo que, en muchos casos, se sopesa a través de la contratación de otra mujer.

A su vez, los hombres, en su mayoría, se consideran ajenos a cualquier tipo de labores domésticas, limitándose al trabajo remunerado y situándose como mantenedor y proveedor del hogar. A la vez, esta situación se ve justificada y legitimada mediante el discurso femenino, que apela a las condiciones culturales, biológicas y hasta estadísticas para explicar la diferencia de roles por género. De esta manera, tal como lo asevera Olavarría (2002), la imagen del hombre como proveedor del hogar que ejerce un trabajo remunerado a tiempo completo, formaría parte del modelo de masculinidad adulta dominante en nuestra sociedad.

Laboreal, Volume $8 \mathrm{~N}^{\circ} 1$ | 2012 
En este contexto, un desafío naciente para el psicólogo laboral - organizacional consiste en la identificación de las prácticas laborales ambiguas e informales, generadoras de menoscabo en la vida de las trabajadoras y desigualdad en las condiciones de trabajo, con el fin de colaborar en la conformación de dinámicas en el entorno laboral más rigurosas, claras y formales, apuntando así a la inserción de la mujer en el mundo del trabajo de manera íntegra y completa, tanto a nivel personal como profesional.

\section{BIBLIOGRAFÍA}

Aguayo, F. (2009). Fronteras del discurso masculino en un Chile globalizado. Reflexiones en torno al relato de hombres profesionales, con pareja y con hijos. Extraído en noviembre, 20, 2011, de http://www.engagingmen.net/files/resources/2010/EME/

2009_fronteras_discurso_masculino_aguayo.pdf

Bonilla-Castro, E, \& Rodríguez, P. (2005). Más allá del dilema de los métodos : la investigación en ciencias sociales. Bogotá : Grupo Editorial Norma.

Burr, V. (1995). An introduction to social constructionism. London : Routledge.

Carrasco, C., \& Mayordomo, M. (2000). Los modelos y estadísticas de empleo como construcción social : la encuesta de población activa y el sesgo de género. Política y Sociedad, 34, 101-112.

Comunidad Mujer (2011a). Mujer y trabajo : los cambios propuestas al sistema de protección de la maternidad. Serie Comunidad Mujer, 10, 1-11.

Comunidad Mujer (2011b). Mujer y trabajo en perspectiva. Serie Comunidad Mujer, 8, 1-11.

Comunidad Mujer (2012). Mujer y trabajo : Servicio doméstico, la ocupación que emplea más mujeres en Chile. Serie Comunidad Mujer, 14, 1-11.

De La Garza, E. (2002). Problemas actuales de la crisis del trabajo. In Eugenia Hola (Ed.), Cambios del trabajo. Condiciones para un sistema de trabajo sustentable (pp. 23 - 31). Santiago : CEM.

Espinoza, V., \& Barozet, E. (2008). ¿De qué hablamos cuando decimos "clase media" ? Perspectivas del caso chileno. Expansiva, 142, 1-35.

Instituto Nacional de Estadísticas (2009). Encuesta exploratoria de uso del tiempo en el gran Santiago. ¿Cómo distribuyen el tiempo hombres y mujeres ? Extraído en diciembre, 16, 2011, de http://estudios.sernam.cl/documentos/?

eMTUwMTY5OA==\%C2\%BFC\%C3\%B3mo_Distribuyen_el_Tiempo_Hombres_y_Mujeres?

Instituto Nacional de Estadísticas (2011). Estadísticas de Empleo. Extraído en enero, 6, 2012, de http://www.ine.cl/canales/chile_estadistico/mercado_del_trabajo/nene/nene.php

Iñiguez, L. (2003). Análisis del discurso. Manual para las ciencias sociales. España : UOC.

Íñiguez, L. (2008). Métodos cualitativos. Extraído en junio, 15, 2010 de http:// psicologiasocial.uab.cat/lupicinio

Maruani, M. (2000). De la Sociología del Trabajo a la Sociología del Empleo. Política y Sociedad, 34, 9-17. 
Maruani, M. (2007). Tiempo, trabajo y género. In Carlos Prieto (Ed.), Trabajo, género y tiempo social (pp. 85-91). España : Hacer - Complutense.

Méndez, M. (2008). Clases medias y ética de la autenticidad. Tensiones en torno al sentido de pertenencia. Extraído en junio, 03, 2012, de http://www.encuesta.udp.cl/descargas/enc2008/07clases-medias-y-etica-de-la-autenticidad.pdf

Ministerio de Desarrollo Social (2009) : Mujeres, encuesta CASEN. Extraído en diciembre, 16, 2011, de http://www.ministeriodesarrollosocial.gob.cl/casen2009/casen_mujeres_2009.pdf

Noguera, J. (2002). El Concepto de Trabajo y la Teoría Social Crítica, Papers, 68, 141-168.

Olavarría, J. (2002). Hombres : identidades, relaciones de género y conflictos entre trabajo y familia. En José Olavarría y Catalina Céspedes (Eds.), Trabajo y familia : ¿conciliación? Perspectivas de género (pp. 53-76). Santiago : SERNAM / FLACSO-Chile / CEM.

Organización Internacional del Trabajo ; Programa de Naciones Unidas para el Desarrollo, (2009). Trabajo y familia : hacia nuevas formas de conciliación con corresponsabilidad social. Extraído en marzo, 14, 2010, de Disponible en : http://www.oit.org.mx/pdf/publicaciones/

trab_fam_resumen.pdf

Prieto, C. (2000). Trabajo y orden social : de la nada a la sociedad de empleo (y su crisis). Política y Sociedad, 34, 19-32.

Prieto, C. (2007). Del estudio del empleo como norma social al de la sociedad como orden social. Papeles CEIC, 28, 1-28.

Programa de Naciones Unidas para el Desarrollo (2010). Desarrollo humano en Chile, género : los desafíos de la igualdad. Extraído en enero, 6, 2012, de http://www.desarrollohumano.cl/ informe-2010/PNUD_LIBRO.pdf

Rau, T. (2011). Flexibilidad de la jornada laboral en Chile : Una tarea pendiente. Extraído en enero, 6, 2012, de http://www.desarrollohumano.cl/informe-2010/PNUD_LIBRO.pdf

Sisto, V., \& Fardella, C. (2009). Control narrativo y gubernamental : la producción de coherencia en las narrativas identitarias. El caso de profesionales chilenos adultos jóvenes en condiciones de vinculación laboral flexible. Extraído en octubre, 10, 2010, de http://www.qualitativeresearch.net/index.php/fqs/article/view/1300/2781

Stecher, A. (2009). El análisis crítico del discurso como herramienta de investigación psicosocial del mundo del trabajo. Discusiones desde América Latina. Universitas Psychologica, 1, 93-107.

Valles, M. (1999), Técnicas cualitativas de investigación social. Madrid : Síntesis.

Yañez, S. (1999). Consideraciones sobre flexibilidad laboral planteadas desde una mirada de género. Extraído en noviembre, 20, 2010, de http://www.cem.cl/pdf/flexibil_laboral.pdf

\section{RESÚMENES}

El presente artículo tiene como objetivo abordar algunas de las problemáticas actuales que afectan a las mujeres chilenas de clase socioeconómica media, residentes en Santiago, en su integración a las dinámicas laborales. Para esto se procesaron entrevistas mediante análisis discursivo, estableciendo tres ejes argumentales : estatus de trabajo y empleo en relación a los riesgos y consecuencias para las mujeres trabajadoras, flexibilidad laboral femenina, y por último, maternidad como comienzo y final de la doble jornada laboral. Los resultados generados muestran que la presencia de factores intra y extra organizacionales afectan las valoraciones 
sociales del trabajo y el empleo ejercido por las mujeres, con una consecuente precarización de estos, debido, en gran parte, a la flexibilización de la jornada laboral. Se aborda el desmedro de la calidad de vida, la justificación que legitima la doble jornada de trabajo, y las tensiones derivadas de tal situación.

Este artigo tem como objectivo abordar algumas das problemáticas atuais que afetam as mulheres chilenas de classe sócio-económica média, residentes em Santiago, na sua integração nas dinâmicas de trabalho. Para isso, foram realizadas entrevistas, cuja análise colocou em evidência três eixos de interpretação : as condições de trabalho e de emprego em relação com os riscos e as consequências para as mulheres trabalhadoras, a flexibilidade do trabalho feminino e, finalmente, a maternidade como início e fim da dupla jornada de trabalho. Os resultados encontrados mostram que a presença de fatores intra e extra organizacionais interferem nas avaliações sociais do trabalho e do emprego exercido pelas mulheres, conduzindo a uma consequente precarização, devido, em grande parte, à flexibilização dos horários. Trata-se de salvaguardar a qualidade de vida, uma justificação que legitima a dupla jornada de trabalho, e as tensões decorrentes desta situação.

Cet article a pour finalité de traiter quelques-unes des questions d'actualité auxquelles sont confrontées les femmes chiliennes, de classe socio-économique moyenne, résidentes à Santiago, lorsqu'elles cherchent à s'intégrer dans la dynamique du travail. Pour ce faire, des entretiens ont été réalisés. L'analyse des retranscriptions a mis en évidence trois axes d'interprétation: les conditions de travail et d'emploi en relation avec les risques et les conséquences pour les femmes, la flexibilité du travail féminin, et enfin, la maternité en tant que début et fin de la double journée de travail. Les résultats montrent que la présence de facteurs intra et extra organisationnels interfèrent dans les évaluations sociales du travail et de l'emploi exercé par les femmes, conduisant à une précarisation, en grande partie en raison d'une flexibilisation des horaires. Il s'agit de sauvegarder une qualité de vie, une justification qui légitime la double journée de travail, et les tensions résultant de cette situation.

This article aims to address some of the current problems affecting socioeconomic middle class Chilean women residents of Santiago, in its integration into the labor dynamics. For this, interviews were processed using discourse analysis, establishing three lines of argument: work and employment status in relation to the risks and consequences for working women, female labor flexibility, and finally, motherhood as beginning and end of the double workday. The results generated show that the presence of intra and extra organizational factors affect social valuations of work and employment exercised by women, with a consequent insecurity of these, due in large part to more flexible working hours. It deals with maintaining the quality of life, the justification that legitimizes the double day of work, and the tensions arising from this situation.

\section{ÍNDICE}

Mots-clés: travail, emploi, flexibilité du travail, maternité

Palavras-chave: trabalho, emprego, flexibilidade laboral, maternidade

Keywords: work, employment, labor flexibility, motherhood

Palabras claves: trabajo, empleo flexibilidad laboral maternidad 


\section{AUTORES}

\section{CONSTANZA GÓMEZ RUBIO}

Becaria CONICYT. Área Psicología del Trabajo y de las Organizaciones, FACSO, Departamento de Psicología, Universidad de Chile. Capitán Ignacio Carrera Pinto 1045, Ñuñoa - Santiago de Chile constagomez@u.uchile.cl

\section{KARINA PÉREZ PLAZA}

Carrera de Psicología, Universidad de Chile, Capitán Ignacio Carrera Pinto 1045, Ñuñoa - Santiago de Chile

kperez@ug.uchile.cl

\section{DENISE DUSSERT CHERVELLINO}

Carrera de Psicología, Universidad de Chile, Capitán Ignacio Carrera Pinto 1045, Ñuñoa - Santiago de Chile

ddussert@ug.uchile.cl 